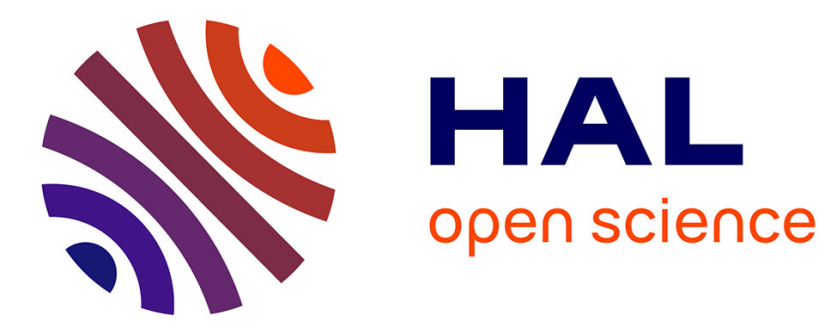

\title{
Stimulated zonal flow generation in the case of TEM and TIM microturbulence
}

Etienne Gravier, Maxime Lesur, Thierry Reveille, Thomas Drouot

\section{To cite this version:}

Etienne Gravier, Maxime Lesur, Thierry Reveille, Thomas Drouot. Stimulated zonal flow generation in the case of TEM and TIM microturbulence. Physics of Plasmas, 2016, 23 (9), pp.92507 - 92507. 10.1063/1.4962845. hal-01489774

\section{HAL Id: hal-01489774 https://hal.science/hal-01489774}

Submitted on 14 Mar 2017

HAL is a multi-disciplinary open access archive for the deposit and dissemination of scientific research documents, whether they are published or not. The documents may come from teaching and research institutions in France or abroad, or from public or private research centers.
L'archive ouverte pluridisciplinaire HAL, est destinée au dépôt et à la diffusion de documents scientifiques de niveau recherche, publiés ou non, émanant des établissements d'enseignement et de recherche français ou étrangers, des laboratoires publics ou privés. 


\title{
Stimulated zonal flow generation in the case of TEM and TIM microturbulence
}

\author{
E. Gravier, M. Lesur, T. Reveille, T. Drouot \\ Institut Jean Lamour, UMR 7198 CNRS - Université de Lorraine \\ 54506 Vandoeuvre-lès-Nancy Cedex, France
}

\begin{abstract}
In this paper we show that in some parameter range in gyrokinetic simulations, it is possible to apply a control method to stimulate the appearance of zonal flows while minimizing the duration of the control process and the impact on plasma parameters. For this purpose, a gyrokinetic code considering only trapped particles is used. The starting point of our work is a situation where zonal flows transiently appear after the nonlinear phase of saturation of TEM (Trapped Electron Modes) or TIM (Trapped Ion Modes) micro-instabilities. These are observed to be strongly reduced in a later phase, permitting streamers to govern the plasma behavior in the steady-state. By intervening during this latter state (after this transient growth and decay of zonal flow), i.e. by increasing the ion/electron temperature ratio for a short time, it is found to be possible to bifurcate to a new steady-state, in which zonal flows are strongly present and are maintained indefinitely, thereby allowing a significant reduction in radial heat fluxes.
\end{abstract}

\section{Introduction}

Zonal flows have attracted the attention of many scientists because of their ability to reduce heat transport $[1,2,3,4,5,6]$. They play an important role in regulating turbulence and hence improve the energy confinement of magnetized plasmas. Zonal flows are fluctuations in the poloidal velocity which are consistently generated by drift wave turbulence through non linear $E \times B$ coupling. Streamers, in contrast, are radially elongated convective cells which dramatically increase radial heat transport in fusion devices. Concerning zonal flows and streamers, several fundamental aspects remain to be fully explored. The mechanisms underlying the onset and disappearance of these structures are still a subject of discussion [7]. In particular the interplay between zonal flows and turbulence remains a challenging problem in tokamak physics [8]. More generally, there is a great deal of interest in studying the competition between streamers and zonal flows, and to enhance strong and robust zonal flows in magnetized plasmas.

The present work is based on a reduced kinetic model for trapped particles $[9,10,11$, $12,13]$. This bounce-averaged gyrokinetic model is a promising tool for investigating fundamental physical processes at low computational cost and good analytic tractability, as a complementary approach to more comprehensive models. Recently, a semi-Lagrangian nonlinear simulation code, TERESA-4D (Trapped Element REduction in Semi lagrangian Approach), has been developed based on this model for trapped ions, making efficient use of parallel computing $[14,15]$. We recently expanded TERESA to simultaneously describe both trapped-ion (TIM) and trapped-electron (TEM) driven modes $[16,17,18]$. The code's most interesting property is that it enables the processing of the full $f$ problem for trapped ions and trapped electrons at very low numerical cost [17]. Competition between streamerlike structures and zonal flows, which is sensitive to the temperature ratio $T_{i} / T_{e}$, has been observed for TEM and TIM turbulence. This is important because zonal flows have been 
shown to play an important role in suppressing nonlinear transport. The impact of the temperature ratio $T_{i} / T_{e}$ on the reduction of zonal flows has been studied [18]. This reduction was found to be linked to the amplitudes of gyro-and-bounce-average density perturbations $n_{e}$ and $n_{i}$ gradually becoming closer.

In this paper we shall show that by intervening after this zonal flow reduction, increasing the ion/electron temperature ratio for a short period of time, it is possible to reach a steadystate in which zonal flows are strongly present, yielding a significant reduction of radial heat fluxes.

The present paper is organized as follows. In Sec. II, the basic bounce-averaged gyrokinetic equations are summarized within the frame of the Hamiltonian description. In Sec. III the main parameters of the simulations are given, as well as the initial and boundary conditions. In Sec. IV the control technique is presented and its impact on the zonal flows is studied in Sec. V. Then, in Sec. VI, the impact of this control on the heat fluxes is discussed. Finally, a brief summary of key results is given along with conclusions.

\section{Model}

In general, trapped particle modes are known to couple with other modes such as ion temperature gradient (ITG) instabilities. However, here, we apply a reduced description, where passing particles are treated adiabatically, and ITG and ETG can not be included. Therefore, the investigations here are meant to uncover qualitative trends.

Our reduced model with trapped kinetic ions and electrons covers both TIM/TEM regimes simultaneously $[16,17]$. The turbulence driven by trapped particles is characterized by frequencies of the order of a slow trapped particle precession frequency. Studying the dynamics at this time scale is therefore relevant for the analysis of TEM and TIM turbulence properties. The study of kinetic trapped particles and adiabatic passing particles makes it possible to average the kinetic equation over the cyclotron and the bounce motions and allows the number of independent variables in phase space to be reduced. The gyro-bounce average filters the fast frequencies $\omega_{c}$ (cyclotron frequency) and $\omega_{b}$ (bounce frequency) and the small space scales $\rho_{c}$ (Larmor radius) and $\delta_{b}$ (banana width). It reduces the dimensionality of the model from $6 \mathrm{D}$ to $4 \mathrm{D}[11,16,17]$.

In this framework the Hamiltonian at the equilibrium only depends on actions which means that kinetic Vlasov equations are much easier to solve. Only two kinetic variables appear in the differential operators. The other two variables appear as parameters - two adiabatic invariants, namely particle kinetic energy $E$ and the trapping parameter $\kappa=\sqrt{\frac{1-\lambda}{2 \varepsilon \lambda}}$, with $\lambda=\mu B_{\text {min }} / E$, and $\varepsilon=\frac{a}{R_{0}}$ the inverse of the aspect ratio of the tokamak. Thus the final model involves two parameters $(E, \kappa)$ and the $2 \mathrm{D}$ space coordinates $(\psi, \alpha)$. The coordinate $\psi$ is the poloidal magnetic flux normalized to $2 \pi$ and stands for the radial coordinate. The quantity $\alpha$ is equal to $\varphi-q \theta$, where $\theta$ and $\varphi$ are respectively poloidal and toroidal angular coordinates and $q$ is the safety factor defined by $q(\psi)=(\mathbf{B} . \nabla \varphi) /(\mathbf{B} \cdot \nabla \theta)$.

More information on the model can be found in [11, 12, 16, 17, 19]. The same assumptions are used hereafter and the model can be summarized as follows.

We make the electrostatic assumption (justified in low- $\beta$ plasmas) and use the MaxwellGauss equation in the quasi-neutral limit (which corresponds to the main turbulence regime in tokamaks). The normalized quasi-neutrality constraint and the evolution of the averaged single particle distribution function for the $s$ species $\bar{f}_{s}(\psi, \alpha, E, \kappa)$ driven by the normalized 


$$
\begin{aligned}
\frac{2}{\sqrt{\pi} n_{e q}}\left(\int_{0}^{+\infty} \mathcal{J}_{0 i} \bar{f}_{i} E^{1 / 2} d E-\int_{0}^{+\infty} \mathcal{J}_{0 e} \bar{f}_{e} E^{1 / 2} d E\right) & =\frac{1}{T_{e q, i}}\left[C_{a d}\left(\Phi-\epsilon_{\phi}\langle\Phi\rangle\right)-C_{p o l} \bar{\Delta}_{i+e}(\Phi)\right] \\
\frac{\partial \bar{f}_{i}}{\partial t}-\left[\mathcal{J}_{0 i} \Phi, \bar{f}_{i}\right]+\Omega_{d} E \frac{\partial \bar{f}_{i}}{\partial \alpha} & =0 \\
\frac{\partial \bar{f}_{e}}{\partial t}-\left[\mathcal{J}_{0 e} \Phi, \bar{f}_{e}\right]-\Omega_{d} E \frac{\partial \bar{f}_{e}}{\partial \alpha} & =0
\end{aligned}
$$

$\Omega_{d}=\frac{Z_{s} \omega_{d}}{E}$, with $Z_{s}$ the charge number of the species $s$, and

$$
\omega_{d}=\frac{2 \mathcal{E}\left(\kappa^{2}\right)}{\mathcal{K}\left(\kappa^{2}\right)}-1+4 s_{0}\left(\frac{\mathcal{E}\left(\kappa^{2}\right)}{\mathcal{K}\left(\kappa^{2}\right)}+\kappa^{2}-1\right)
$$

where $s_{0}$ is the magnetic shear and $\mathcal{K}\left(\kappa^{2}\right)$ and $\mathcal{E}\left(\kappa^{2}\right)$ are respectively the complete elliptic functions of the first and second kinds. The gyro-bounce-average operator $\mathcal{J}_{0 s}$ can be written as :

$$
\mathcal{J}_{0 s}=\left(1-\frac{E}{T_{e q, s}} \frac{\delta_{b 0, s}^{2}}{4} \partial_{\psi}^{2}\right)^{-1}\left(1-\frac{E}{T_{e q, s}} \frac{q^{2} \rho_{c 0, s}^{2}}{4 a^{2}} \partial_{\alpha}^{2}\right)^{-1}
$$

with $\rho_{c 0, s}$ and $\delta_{b 0, s}$ the Larmor radius and the banana width (expressed in units of $\psi$ ) at an arbitrary typical temperature $T_{0}$ (energy is normalized to the typical thermal energy $\left.T_{0}\right), \delta_{b 0, s}=q \rho_{c 0, s} / \sqrt{\epsilon}, q$ the safety factor and $\varepsilon$ the inverse of the aspect ratio, and $T_{e q, s}$ the equilibrium temperature of the species $s$. The Poisson's brackets are defined by $[f, g]=$ $\partial_{\alpha} f \partial_{\psi} g-\partial_{\alpha} g \partial_{\psi} f$ and $C_{p o l}=\frac{e \omega_{0} L_{\psi}}{T_{0}}, C_{a d}=\frac{C_{p o l}\left(1-f_{T}\right)}{f_{T}}(1+\tau)$ et $\bar{\Delta}_{i+e}(\Phi)=\bar{\Delta}_{i}(\Phi)+\tau \bar{\Delta}_{e}(\Phi)$, with $f_{T}=\frac{2 \sqrt{2 \varepsilon}}{\pi}$ the fraction of trapped particles, $\tau=\frac{T_{e q, i}}{T_{e q, e}}, \omega_{0}=T_{0} /\left(e R_{0}^{2} B_{\theta}\right)$ which corresponds to the ion precession frequency at the typical temperature $T_{0}$ (time is normalized to this frequency), $L_{\psi}=a R_{0} B_{\theta}$ the length of the simulation box in $\psi$, and $\bar{\Delta}_{s}$ the operator defined by :

$$
\bar{\Delta}_{s}=\left(\frac{q \rho_{c 0, s}}{a}\right)^{2} \partial_{\alpha}^{2}+\delta_{b 0, s}^{2} \partial_{\psi}^{2}
$$

with $a$ the small radius. $\epsilon_{\phi}=\frac{\epsilon_{\phi, i}+\tau \epsilon_{\phi, e}}{1+\tau}$ is a control parameter which governs the response of the adiabatic passing particles. It depends on the responses of the passing ions $\epsilon_{\phi, i}$ and the passing electrons $\epsilon_{\phi, e}$.

Regarding the electron response, $\epsilon_{\phi, e}=1$ prevents the passing electrons from responding to the zonal potential which is constant on a flux surface (toroidal mode $n=0$ ). This situation prevails when the electron gyro radius is small compared with the characteristic length of the zonal flows [6]. $\langle\Phi\rangle=\langle\Phi\rangle_{\alpha}=\Phi_{Z F}$ stands for the electrostatic potential averaged on the flux surface, i.e. the zonal potential. Unlike passing electrons, passing ions must respond to zonal flows $\left(0 \leq \epsilon_{\phi, i} \leq 1\right)$, therefore $\epsilon_{\phi}$ should be in the range $[0,1]$. The model does not allow the adiabatic response of the passing particles to be properly computed so we introduce $\epsilon_{\phi}$ as a free parameter, $0 \leq \epsilon_{\phi} \leq 1$ and assume the form chosen in Eq.1a. A detailed study of this parameter was performed in [15]. Hereafter only the extreme case $\epsilon_{\phi}=1$ simulations will be used in the paper.

Using the quasi-neutrality equation (1a) and expressing the zonal potential as a function of electron and ion density fluctuations, we obtain

$$
\Phi_{Z F} \sim \frac{\left\langle n_{i}\right\rangle_{\alpha}-\left\langle n_{e}\right\rangle_{\alpha}}{C_{a d} n_{0}\left(1-\epsilon_{\phi}\right)+C_{p o l} k^{2}\left(\delta_{b, i}^{2}+\tau \delta_{b, e}^{2}\right) n_{0}}
$$

with $k$ the radial wavenumber derived from the Laplacian operator $\bar{\Delta}_{s}$ in Fourier space, $n_{0}$ the density reference at equilibrium. $n_{s}$ denotes the perturbed part of the density. For close 
values of $C_{a d}$ and $C_{p o l}$ which is the main case we study in the rest of this work and with $\epsilon_{\phi}=1$, the zonal potential can be written as:

$$
\Phi_{Z F} \sim \frac{1}{n_{0} C_{p o l}} \frac{\left\langle n_{i}\right\rangle_{\alpha}-\left\langle n_{e}\right\rangle_{\alpha}}{k^{2} \delta_{b, \mathrm{eff}}^{2}}
$$

where we define the effective banana width by $\delta_{b, \text { eff }}^{2}=\delta_{b, i}^{2}+\tau \delta_{b, e}^{2}$. Finally the electric potential shearing rate can be derived from this latter equation and written as:

$$
\partial_{\psi}^{2} \Phi_{Z F} \sim k^{2} \frac{\left\langle n_{i}\right\rangle_{\alpha}-\left\langle n_{e}\right\rangle_{\alpha}}{C_{p o l} k^{2} \delta_{b, \mathrm{eff}}^{2} n_{0}} \sim \frac{\left\langle n_{i}\right\rangle_{\alpha}-\left\langle n_{e}\right\rangle_{\alpha}}{C_{p o l} \delta_{b, \mathrm{eff}}^{2} n_{0}}
$$

\section{$3 \quad$ Numerical simulations}

The gyrokinetic code developed is named TERESA-4D [17] (Trapped Element REduction in Semi lagrangian Approach). It currently runs in a 4D electrostatic version for $N$ kinetic trapped species including the electrons. Passing particles are assumed to be adiabatic. In order to solve the Vlasov equations (Eq.1b and 1c), the code takes advantage of two approaches [20], namely a semi-Lagrangian scheme using a fixed grid (Eulerian) while also taking into account the conservation of the value of the distribution function along a trajectory in phase space (Lagrangian). To solve the electro-neutrality (Eq.1a), the fields are first projected in the Fourier space along the periodic direction $\alpha$. The electric potential $\Phi$ is then a solution of a second order differential equation in $\psi$. The latter is solved up to fourth order in $\psi$ by inverting a penta-diagonal matrix.

This code is full $f$ : the whole distribution function is computed. To study heat transport, a transition region where the fluctuations are damped towards their prescribed vanishing value at the edge was set up.

A buffer region enforces this transition layers. It is controlled by an ad hoc diffusion coefficient $D$. The right-hand side of equations (1b and 1c) then reads $\partial_{\psi}\left(D \partial_{\psi} \bar{f}_{s}\right)$. The diffusion coefficient is non-vanishing in the buffer regions only $(0 \leq \psi \leq 0.05$ and $0.95 \leq$ $\psi \leq 1$ ), where its maximum value is $10^{-2}$. The lengths are given in $L_{\psi}$ units. It is given a shape [12] which means that the buffer regions occupy less than $10 \%$ of the radial domain of the simulation box.

Temperature and density gradients are described by $\kappa_{n}=0$ and $\kappa_{T}=0.25$, with $\kappa_{n, T}=\partial_{\psi} \log \left(n_{e q}, T_{e q}\right)$. The equilibrium temperature is allowed to change so that the final equilibrium is self-consistently computed. A thermal bath is used with the temperature fixed on the radial boundaries. Dirichlet boundary conditions are imposed on the electrostatic potential at $\psi=0$ and $\psi=1$. The time step chosen for TEM/TIM simulations performed with TERESA is typically $\Delta t=0.01$ (in $\omega_{0}^{-1}$ units). The equilibrium distribution functions are given the form:

$$
F_{e q, s}=\frac{n_{s}(\psi)}{T_{s}^{\frac{3}{2}}(\psi)} \exp \left(-\frac{E}{T_{s}(\psi)}\right)
$$

The temperature writes:

$$
T_{s}=T_{e q, s}+\frac{\kappa_{T, s}}{2}\left(1+L_{T}\left[\log \left(\cosh \left(\frac{\psi-\Psi_{1}}{L_{T}}\right)\right)-\log \left(\cosh \left(\frac{\psi-L_{\psi}+\Psi_{1}}{L_{T}}\right)\right)\right]\right)
$$

with $L_{T}=0.025, \Psi_{1}=0.1 . \quad \kappa_{T}$ is almost constant over the entire radial width of the simulation box except in the buffer regions where $\kappa_{T}=0$. A constant equilibrium density profile is chosen and can be expressed as: $n_{s}=n_{e q, s}=n_{0}=1$. 
The parameters given in Tab.1 enable us to study modes with toroidal mode number $n$ in the range $[0,128]\left(N_{\alpha}=257\right)$ with the shortest wavelengths being of the order of $\rho_{c, i}$. The grid mesh is $N_{\psi}=257$ in the radial direction and $N_{E}=96$ for the energy parameter. The trapping parameter $\kappa$ and the magnetic shear $s_{0}$ are equal to zero.

\begin{tabular}{cccccccccccccccc}
\hline$\kappa_{n_{i, e}}$ & $\kappa_{T_{i, e}}$ & $\delta_{b, i}$ & $\delta_{b, e}$ & $\rho_{c, i}$ & $\rho_{c, e}$ & $C_{p o l}$ & $C_{a d}$ & $\Omega_{d}$ & $\frac{T_{e q_{i}}}{T_{e q_{e}}}$ & $N_{\psi}$ & $N_{\alpha}$ & $N_{E}$ & $\epsilon_{\phi}$ & $\kappa$ & $s_{0}$ \\
\hline 0.0 & 0.25 & 0.1 & 0.01 & 0.03 & 0.01 & 0.1 & 0.05 & 1 & 0.5 & 257 & 257 & 96 & 1 & 0 & 0 \\
\hline
\end{tabular}

Table 1: The main parameters used for the nonlinear simulations, in the case $T_{e}=2 T_{i}$.

\section{Control method}

The control method proposed in this paper is to temporarily increase the ion/electron temperature ratio $\tau$. This can be interpreted as a crude model for a variety of experimental scenarios:

1. A temporary decrease in ECRH (Electron Cyclotron Resonance Heating). ECRH is a powerful heating tool with which heating energy may be deposited at selected locations, thus tailoring the temperature profile in the plasma. ECRH is used in many tokamaks - the ITER Tokamak will rely on ECRH heating - and is also for instance the main heating system of Wendelstein 7-X, capable to operate continuously. When ECRH heating is switched on in experimental devices it takes between 10 to $100 \mathrm{~ms}$ for the wave to transfer its energy to the electrons which corresponds to a $10-100 \omega_{0}^{-1}$ range in our simulations.

2. A temporary increase in ICRH (Ion Cyclotron Resonance Heating). By placing an antenna very close to the surface of the plasma it is possible to heat the ions at the resonant cyclotron frequencies of the ions. ICRH can produce a strong absorption of energy at the center of the plasma. It is produced in many present day tokamaks. It will be one of the heating systems installed in ITER and it will deliver $20 \mathrm{MW}$ of radio frequency power to the plasma.

3. A temporary increase in NBI (Neutral Beam Injection). Neutral beam injection consists in injecting high-energy neutral particles into the plasma. They are unaffected by the magnetic field and travel along straight-line trajectories until they are ionized by collisions with the plasma. The new high-energy tail on the distribution function (deuterium particles for instance) is then slow down by Coulomb collisions and transfers its energy to the background plasma in the form of heat. This heating method will also be used in ITER.

Starting from a situation where $T_{e}=2 T_{i}$, we observed that zonal flows occur at the beginning of the nonlinear phase and then are reduced allowing for streamers to govern heat radial transport. We decide to stop the electron heating $\left(T_{e}=T_{i}\right)$ for a short period of time $\left(\tau_{c}+2 t_{r}\right.$, see Fig.1, with $t_{r}$ the time necessary to switch the heating on or off and $\tau_{c}$ the time during which the electron temperature is reduced and maintained constant) and then to heat the electrons again $\left(T_{e}=2 T_{i}\right)$ if possible with the aim of obtaining strong and robust zonal flows. Indeed, as we shall describe in the following sections, we observed that zonal flows might be strong and maintained indefinitely in the nonlinear phase when temperatures are equal. Our idea is thus to try to apply this short disruption to trigger strong zonal flows and we expect these would be maintained even when the electron heating to $T_{e}=2 T_{i}$ is 
attained once again. It should be noticed here that the control method is not modified by feedback and does not depend on the behavior of the dynamical system as could be the case for control methods used for chaos control [21, 22].

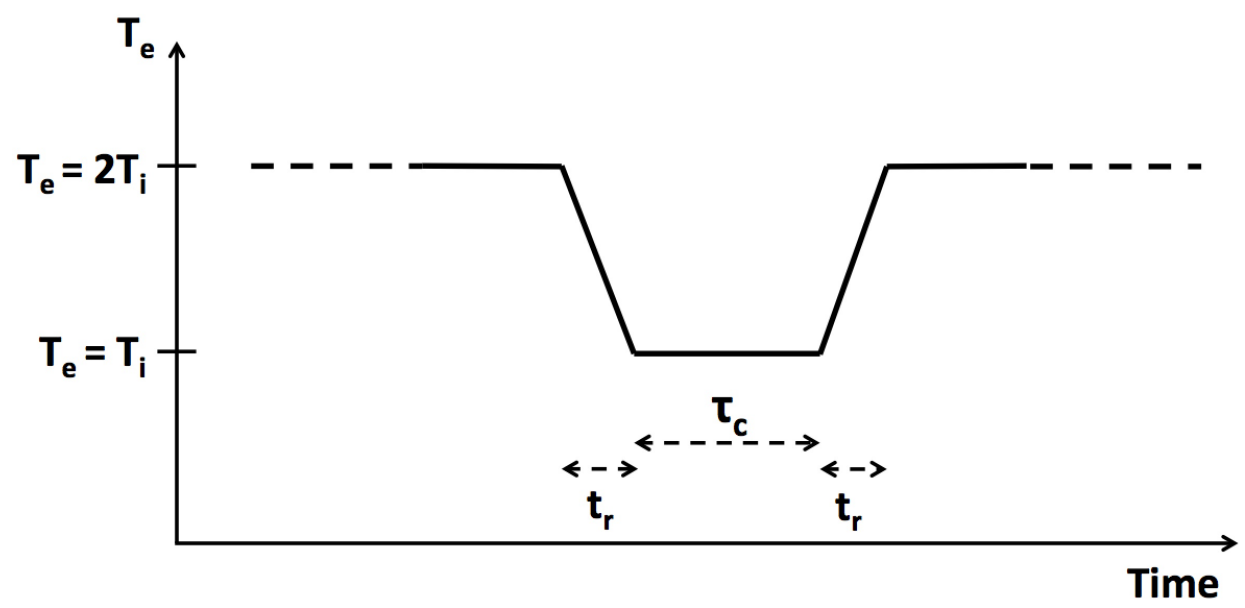

Figure 1: Principle of the control method. Electron heating is switched off during a short time $\tau_{c}$, and then switched on again. $t_{r}$ is the time necessary to reduce or increase the electron temperature.

We experimented with the following three ways to act on $T_{i} / T_{e}$ :

- Method M1: modifying the $C_{a d}$ coefficient (see Eq.1), which is a function of the temperature ratio.

- Method M2c: modifying the electron temperature of the thermal bath in the core side.

- Method M2e: modifying the electron temperature of the thermal bath in the edge side.

It should be noted that we found the qualitative response of the plasma (which will be discussed in sections 5 and 6 ) to be insensitive to the duration of the control period during which we modify the plasma parameters, as both durations $\tau_{c}+2 t_{r}=0.26 \omega_{0}^{-1}$ and $\tau_{c}+2 t_{r}=10 \omega_{0}^{-1}$ lead to the same results for instance. Moreover, whatever the duration needed to switch the electron heating on or off (very rapid: $t_{r}=0.01 \omega_{0}^{-1}$, or gradual: $\left.t_{r}=10.0 \omega_{0}^{-1}\right)$, we obtain the same results regarding the emergence of zonal flows.

Finally, we have observed that, for $T_{e}=2 T_{i}$, the efficiency of the control is not very sensitive to the applied method. In general, zonal flows can be triggered regardless of the choice of method, M1, M2c or M2e. Nevertheless, for instance in the case of $T_{e}=3 T_{i}$, for which the zonal flows are very weak without control, it has been observed that strong zonal flows are recovered by temporarily modifying the thermal baths (M2c and M2e), whereas modifying $C_{a d}$ alone (M1) is no longer an efficient method. In the rest of the paper the $T_{e}=$ $2 T_{i}$ case is presented in detail with a control method focused on method M1 (modification of the $C_{a d}$ coefficient) during $\tau_{c}+2 t_{r}=0.26 \omega_{0}^{-1}$.

\section{Stimulate zonal flow generation}

For this paper we study the $T_{e}=2 T_{i}$ case and we describe in detail the effects of the control method applied in this case. For the set of parameters given in Tab.1 we compare the case with no control, to the case with a control applied between $t=20$ and $t=20.26 \omega_{0}^{-1}$, meaning that the electron heating is switched off $\left(T_{e}=T_{i}, C_{a d}=0.1\right.$, method M1) during this short period of time $\left(t_{r}=0.01 \omega_{0}^{-1}, \tau_{c}=0.24 \omega_{0}^{-1}\right)$ before being switched back on. 


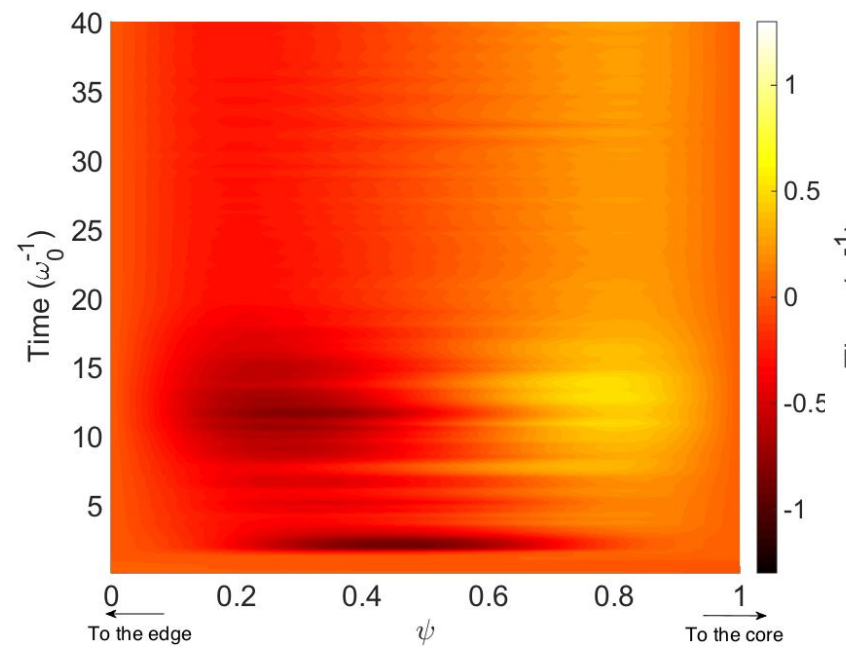

(a)

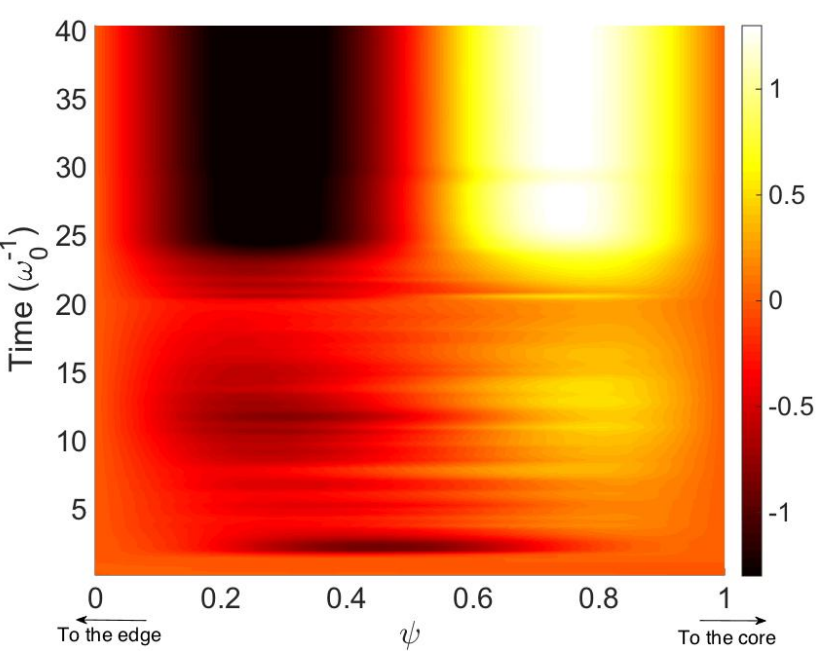

(b)

Figure 2: Zonal potential $\Phi_{\mathrm{ZF}}$ (electric potential averaged over $\alpha$ ) as a function of $\psi$ and time for $T_{e}=2 T_{i}$. For this set of parameters given in Tab.1 the simulation exhibits TIMdominant instabilities and streamers in its linear phase, then zonal flows and streamers in its nonlinear phase. (a) and (b) are two different simulations with the same input parameters and same initial conditions. The only difference is that in (a) no control was applied, while in (b) the control method M1 was applied between $t=20$ and $t=20.26 \omega_{0}^{-1}$.

Figure 2 shows the behavior of the averaged potential on $\alpha,\langle\Phi\rangle_{\alpha}=\Phi_{Z F}$, as a function of $\psi$ and time. It should be noted that instabilities and streamer structures are found to develop in the linear phase according to the most unstable linear TIM mode $\left(k_{\psi}=\pi / L_{\psi}\right.$, $n=13)$. Then, nonlinear mode coupling makes these structures coalesce, which leads to a saturated state dominated by zonal flows. The strongest zonal flows seem to occur at $t=11.4 \omega_{0}^{-1}$ and then are reduced (see Fig.2 (a)).

As we have observed zonal flows to be strong and robust in the $T_{i} / T_{e}=1$ case, we try to apply this temperature ratio to the system between $t=20$ and $t=20.26 \omega_{0}^{-1}$. After this time the temperature ratio is brought back up to its previous value $T_{i} / T_{e}=0.5$. The results are given in Fig. 2 (b). Of course the behavior of the plasma is the same from $t=0$ to $t=20$ $\omega_{0}^{-1}$ but next, after a short transition after the end of the control, the system is again found to exhibit strong zonal flows. The amplitude of these zonal flows is almost constant after $t=25 \omega_{0}^{-1}$, and until we arbitrarily end the simulation (we have checked up to $t=250 \omega_{0}^{-1}$ ). Therefore, robust and strong zonal flows appear to be triggered by the applied control.

This behavior can also be seen in Fig. 3 where the electric potential shearing rate profile is plotted against $\psi$ for both cases (with and without control) and for two different times (at $t=11.4 \omega_{0}^{-1}$, dotted line and at $t=30.0 \omega_{0}^{-1}$, solid line). At $t=11.4 \omega_{0}^{-1}$, before the control method is applied, the electric potential shearing rate is of course the same for Fig. 3 (a) and (b). However, as we can note, the zonal flows at $t=30.0 \omega_{0}^{-1}$ recovered with the control method (Fig. 3 (b), solid line) are much stronger than those observed at the same time without any control (Fig. 3 (a), solid line). At $\psi=0.3$ and $\psi=0.7$, where control-induced zonal flows peak, the shearing rate at $t=30 \omega_{0}^{-1}$ is an order-of-magnitude larger with control than without. Clearly the control method is very effective throughout most of the domain, and therefore a high level of improvement in energy confinement can be expected globally.

We observe in Fig. 3 (b) that the amplitude of the zonal flows obtained at $t=30.0 \omega_{0}^{-1}$ is about twice as large as that of observed at $t=11.4 \omega_{0}^{-1}$. According to the equation (7), 


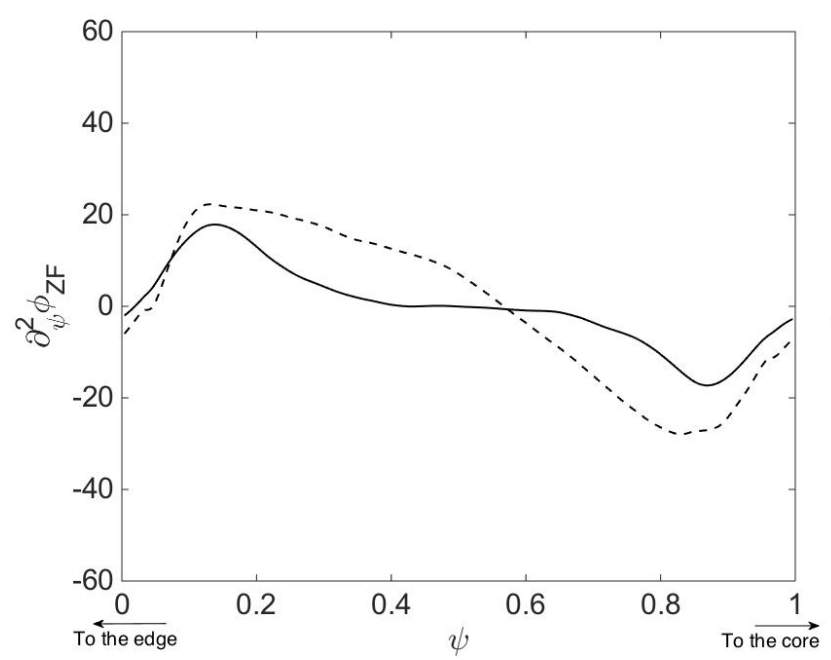

(a)

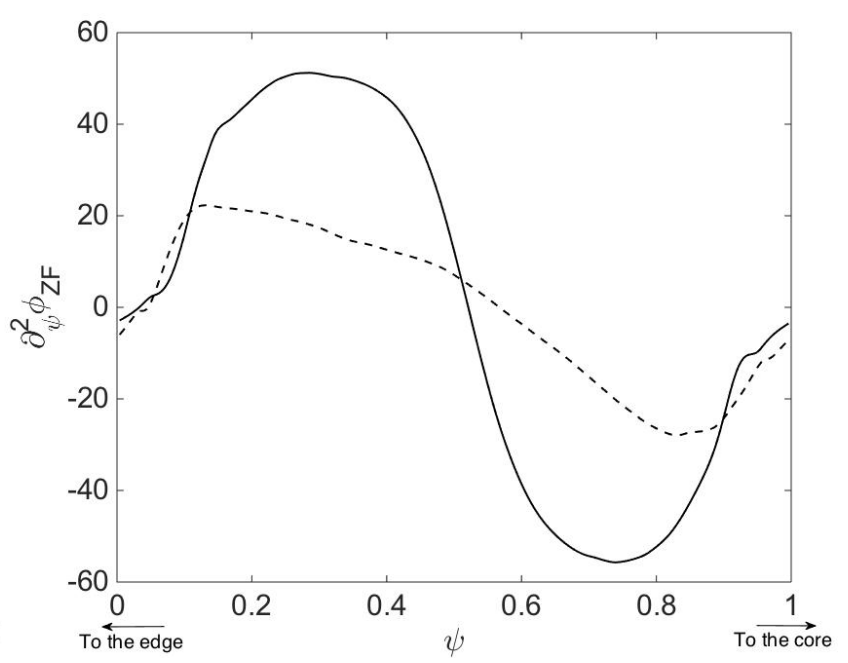

(b)

Figure 3: Electric potential shearing rate profile averaged over $\alpha$ as a function of $\psi$, at $t=11.4 \omega_{0}^{-1}$ (dotted line) and at $t=30.0 \omega_{0}^{-1}$ (solid line) in the case with no control (a) and in the case where control was applied between $t=20$ and $t=20.26 \omega_{0}^{-1}$ (b).

this factor two should also be recovered in the density difference $\left|<n_{i}\right\rangle_{\alpha}-\left\langle n_{e}\right\rangle_{\alpha} \mid$, since the electric potential shearing rate is proportional to this difference.

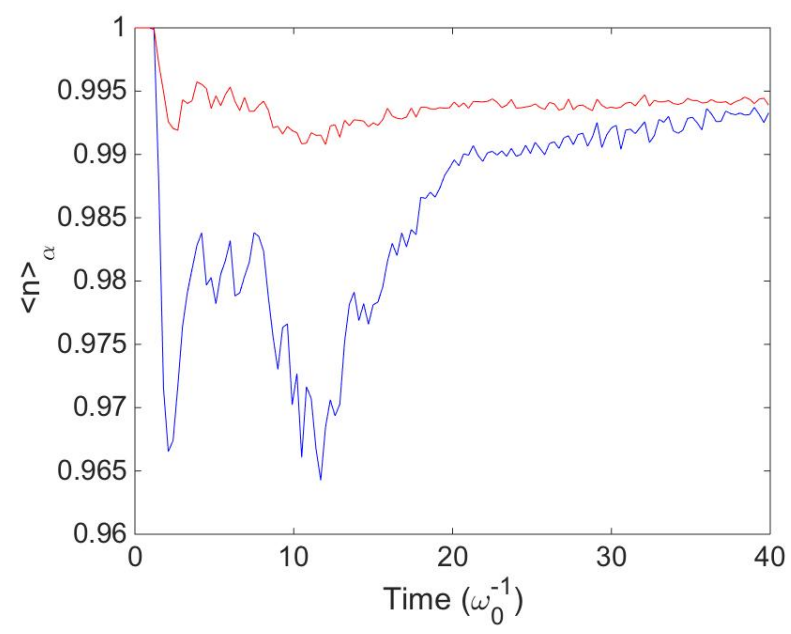

(a)

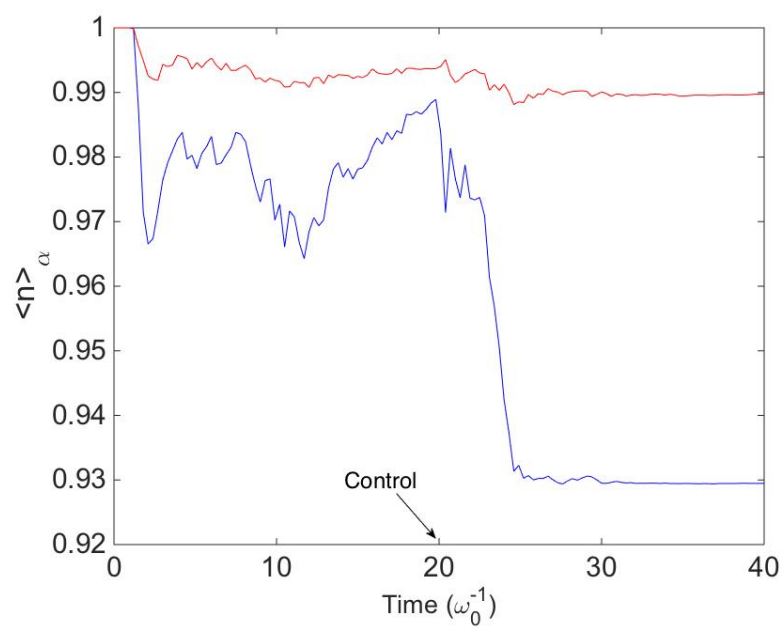

(b)

Figure 4: Ion and electron gyro-and-bounce average densities plotted against time. Ion density is in blue, electron density is in red. In (a) is plotted the case without control and in (b) the case with the control applied.

To check this, the time evolution of ion (in blue) and electronic (in red) gyro-and-bounce average densities are drawn Fig. 4 , at $\psi=0.39$. First it should be noted that the ion density is found to have a larger amplitude response than the electron density since TIM rather than TEM are linearly destabilized with the set of parameters used for the simulation. Fig. 4 also shows that the ions appear to evolve to adjust themselves to the level of electronic fluctuation and thus is found to govern the dynamics of zonal flows. We observe Fig.4 (b) that the density difference at $t=30.0 \omega_{0}^{-1}$ is about twice as large as that observed at $t=11.4$ $\omega_{0}^{-1}$. As expected this result is in agreement with equation (7) and the amplitude of the zonal flows observed previously in Fig.3 (b). We also noticed that the large density difference is in agreement with the results obtained in a previous study in which a strong steady zonal- 
flow regime is correlated with a large density difference in the case $T_{e}=T_{i}$ (See Fig.5a in [18]). The species that is involved in the instability which drives turbulence acts the most in response to the zonal potential and separates from (adjusts to) the fluctuation level of the other species which then leads to the zonal flow amplification (reduction). This could explain the dependency of the stimulation mechanism with respect to $\tau$. Indeed Ref.[18] shows a natural transition from zonal flow-dominated regime to streamer-like regime as $T_{i} / T_{e}$ decreases. Therefore it can be expected that stimulation is only efficient at relatively large temperature ratio.

Finally, focusing here on the densities, we again observe that the control applied between $t=20$ and $t=20.26 \omega_{0}^{-1}$ is found to be sufficient to generate and subsequently maintain a significant difference between electron and ion densities which allows the strong zonal flows to govern plasma behavior. It should be noted here that the actual mechanism of the transition remains unexplained and further investigations are kept for future work.

\section{Impact on the heat fluxes}

Heat transport from the hot plasma core towards the colder plasma boundary is highly connected to the turbulence level and the competition between zonal flows and streamers. Our aim is to reduce heat fluxes by stimulating zonal flows. In our model, the heat conductive flux $q_{s}$ is related to the heat flux $Q_{s}$ by [12]:

$$
q_{s}=Q_{s}-\Gamma_{s} T_{s}
$$

with $q_{s}=-n_{s} \chi_{s} \partial_{\psi} T_{s}, Q_{s}=-\frac{2}{\sqrt{\pi}} \int d \alpha \int d E E^{3 / 2} \partial_{\alpha}\left(J_{0 s} \Phi\right) f_{s}$ and the guiding centre flux $\Gamma_{s}=-\frac{2}{\sqrt{\pi}} \int d \alpha \int d E E^{1 / 2} \partial_{\alpha}\left(J_{0 s} \Phi\right) f_{s}$.

Figure 5 shows the conductive heat flux $q_{s}$ averaged over $\alpha$ and $\psi$, as a function of time. The effect of the control on the heat flux is clearly noticeable. After the control was applied and after a transition period of about $5 \omega_{0}^{-1}$, the system stabilizes and reaches a steady state in which the heat flux is reduced.

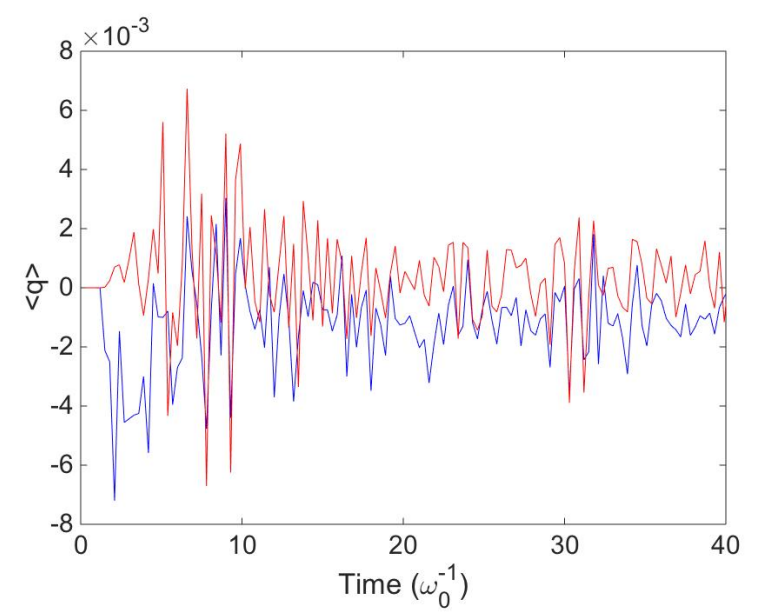

(a)

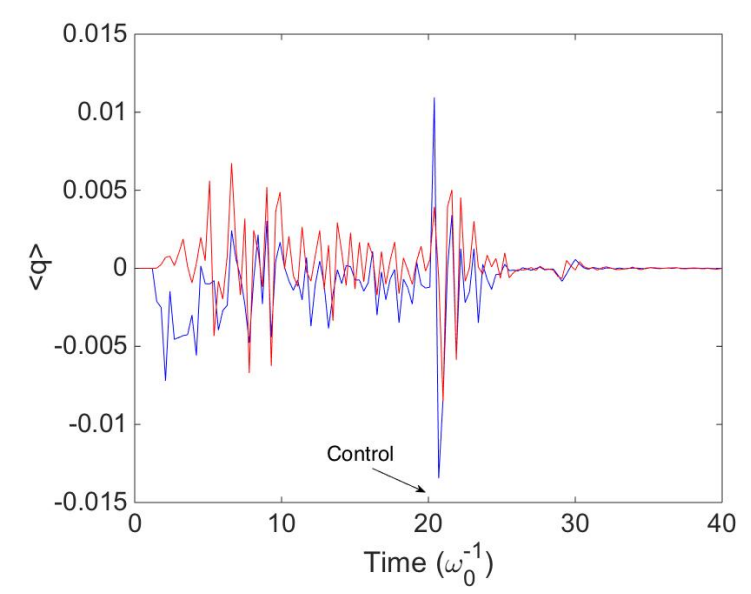

(b)

Figure 5: Conductive electron heat flux $q_{e}$ (in red) and conductive ion heat flux $q_{i}$ (in blue) as a function of time. $q_{e}$ and $q_{i}$ are averaged over $\alpha, \psi$. In (a) the case without control is plotted and (b) shows the case with control applied.

To quantify this effect, the ion heat flux is averaged over time from $t=25$ to $t=40 \omega_{0}^{-1}$. In the case without control, $\left\langle q_{i}\right\rangle_{t}$ is found to be equal to $-10^{-3}$, whereas in the case with 
control $\left\langle q_{i}\right\rangle_{t}$ is strongly reduced as expected and is equal to $-6.5 \times 10^{-5}$ thus proving the efficiency of the control method in reducing the radial heat transport. As expected streamers are found to lead to a large conductive heat flux while turbulent transport is greatly reduced in the presence of zonal flows. Here the heat transport is mainly carried by the trapped ions and transported outwards (downgradient, note that in our model $\psi$ is proportional to $-r$ ).

Although, for the sake of concision, we have focused on one set of parameters and one control method, we have observed qualitatively similar results (control-induced zonal flows with strong reduction in ion heat flux), for a range of parameters and different control methods. Table 2 lists the cases that we have tested, and for each case, whether a significant and robust bifurcation to zonal-flow-dominated steady-state was found $(\mathrm{Y})$ or not $(\mathrm{N})$.

\begin{tabular}{|c|c|c|c|c|c|c|c|c|c|c|c|c|c|}
\hline Control method & \multicolumn{6}{|c|}{ M1 } & \multicolumn{4}{|c|}{ M2c } & \multicolumn{3}{|c|}{ M2e } \\
\hline$t_{r}\left[\omega_{0}^{-1}\right]$ & \multicolumn{4}{|c|}{0.01} & 0.1 & 10 & \multicolumn{4}{|c|}{10} & \multicolumn{3}{|c|}{10} \\
\hline$\tau_{c}\left[\omega_{0}^{-1}\right]$ & \multicolumn{5}{|c|}{0.24} & 10 & \multicolumn{4}{|c|}{0} & \multicolumn{3}{|c|}{0} \\
\hline Initial $T_{i} / T_{e}$ & 0.57 & 0.5 & 0.4 & 0.33 & 0.5 & 0.5 & 0.5 & & .4 & 0.33 & 0.5 & 0.4 & 0.33 \\
\hline Control $T_{i} / T_{e}$ & \multicolumn{6}{|c|}{1.0} & 1.0 & 1.0 & 0.67 & 1.0 & 1.0 & 1.0 & 1.0 \\
\hline Bifurcation & $\mathrm{Y}$ & Y & $\mathrm{N}$ & $\mathrm{N}$ & Y & $\mathrm{Y}$ & Y & Y & $\mathrm{Y}$ & $\mathrm{Y}$ & $\mathrm{Y}$ & $\mathrm{Y}$ & $\mathrm{Y}$ \\
\hline
\end{tabular}

Table 2: List of cases that we have simulated. The last line, "Bifurcation", indicates for each case, whether we observe, yes $(\mathrm{Y})$ or no $(\mathrm{N})$, after the control, robust and strong zonal flows along with significantly reduced ion heat flux.

\section{Conclusion}

To sum up, we investigated the possibility of stimulating zonal flow generation. Zonal flows are driven by TEM and TIM turbulence and studied by means of gyrokinetic Vlasov simulations for trapped particles. We have shown that in cases where zonal flows normally appear only transiently at the beginning of a simulation it is possible to trigger a bifurcation from a standard steady-state dominated by radially-elongated structures, to a new steadystate dominated by zonal flows, by shortly increasing the $T_{i} / T_{e}$ ratio. After a short transition, zonal flows appear again and are sustainable and very effective regarding the reduction of heat fluxes.

When effective, the control method has been demonstrated to prevent densities becoming similar thus making it possible to maintain a finite radial electric field after the control was applied. The advantage of this method is that plasma parameters are modified only for a very short period of time without significantly modifying the performance of the plasma on the discharge time and while allowing the plasma to be heated again indefinitely.

Several numerical models for the control method were tested. It is possible to modify the plasma behavior by changing the $C_{a d}$ coefficient which is a function of the electron temperature or by modifying the boundary conditions i.e. the temperatures of the thermal bath or both. We also observed that the plasma dynamics is not affected by the duration of the control applied.

However, we also noticed that this method is only effective for a certain range of temperatures. Indeed, the reduction phenomenon of the zonal flows varies in importance and 
gradually evolves depending on the ratio $T_{i} / T_{e}$. For instance for $T_{e}=3 T_{i}$, zonal flows are more strongly reduced than for $T_{e}=2 T_{i}$ and we also observed that in this case the method is efficient only when modifying boundary conditions and thermal bath conditions whereas modifying $C_{a d}$ alone is found to have no lasting effect on plasma behavior. The greater the reduction of zonal flows during the nonlinear phase of the instabilities, the more it is difficult for the zonal flows to be stimulated by the control method.

Further study will be required to investigate and find out what the precise nonlinear saturation mechanism is. Such mechanisms are found in the literature - for instance KelvinHelmholtz instabilities and vortices could play an important role in the saturation and decay of streamers by merging to produce zonal flows [23]. Moreover, the close correlation between these structures, which gradually disappear to once again produce streamers, and the evolution of the ion density which gets very close to the electron density also remains to be explained. These studies should help us to understand why the control method carried out in this numerical experiment is efficient. We shall therefore leave this question for future work.

\section{Acknowledgments}

This work was carried out within the framework of the European Fusion Development Agreement and the French Research Federation for Fusion Studies. The views and opinions expressed herein do not necessarily reflect those of the European Commission.

\section{References}

[1] Z. Lin, T. S. Hahm, W. W. Lee, W. M. Tang and R. B. White, Turbulent transport reduction by zonal flows: Massively parallel simulations, Science 281, 1835 (1998)

[2] B. N. Rogers, W. Dorland, and M. Kotschenreuther, Generation and Stability of Zonal Flows in Ion-Temperature-Gradient Mode Turbulence, Phys. Rev. Lett. 85, 5336 (2000)

[3] E. Kim and P. H. Diamond, Dynamics of zonal flow saturation in strong collisionless drift wave turbulence, Phys. Plasmas 9, 4530 (2002)

[4] P. H. Diamond, S. I. Itoh, K. Itoh and T. S. Hahm, Zonal flows in plasma, Plasma Phys. Control. Fusion 47, R35 (2005)

[5] Y. Asahi, A. Ishizawa, T. H. Watanabe, H. Tsutsui, S. Tsuji-lio, Regulation of electron temperature gradient turbulence by zonal flows driven by trapped electron modes, Phys. Plasmas 21, 052306 (2014)

[6] X. Garbet, Y. Idomura, L. Villard and T.H. Watanabe, Gyrokinetic simulations of turbulent transport, Nucl. Fusion 50, 043002 (2010)

[7] Z. B. Guo, T. S. Hahm, Zonal flow generation and its nonlinear dynamics in trapped electron mode turbulence of flat density tokamak plasmas, Nucl. Fusion 56, 066014 (2016)

[8] G. R. Tynan, I. Cziegler, P. H. Diamond, M. Malkov, A. Hubbard, J. W. Hugues, J. L. Terry and J. H. Irby, Recent progress towards a physics-based understanding of the H-mode transition, Nucl. Fusion 56, 066014 (2016)

[9] M. Tagger, G. Laval, and R. Pellat, Nucl. Fusion 17, 109 (1977). 
[10] H. Biglari, P. H. Diamond, and P. W. Terry, Phys. Fluids 31, 2644 (1988).

[11] G. Depret, X. Garbet, P. Bertrand, A. Ghizzo, Trapped-ion driven turbulence in tokamak plasmas Plasma Phys. Cont. Fusion 42, 949 (2000)

[12] Y. Sarazin, V. Grandgirard, E. Fleurence, X. Garbet, Ph. Ghendrih, P. Bertrand and G. Depret, Kinetic features of interchange turbulence, Plasma Phys. Control. Fusion 47, 1817 (2005)

[13] G. Darmet, Ph. Ghendrih, Y. Sarazin, X. Garbet, V. Grandgirard, Intermittency in flux driven kinetic simulations of trapped ion turbulence, Commun. Nonlinear Sc. Numerical Simulation 13, 53 (2008)

[14] T. Cartier-Michaud, P. Ghendrih, V. Grandgirard, and G. Latu, ESAIM: Proc. 43, 274 (2013)

[15] T. Cartier-Michaud, P. Ghendrih, Y. Sarazin, G. Dif-Pradalier, T. Drouot, D. Estève, X. Garbet, V. Grandgirard, G. Latu, C. Norscini, C. Passeron, J. Phys.: Conf. Series $561,012003(2014)$

[16] T. Drouot, E. Gravier, T. Reveille, A. Ghizzo, P. Bertrand, X. Garbet, Y. Sarazin and T. Cartier-Michaud, A gyro-kinetic model for trapped electron and ion modes, Eur. Phys. Journal D 68, 280 (2014)

[17] T. Drouot, E. Gravier, T. Reveille, M. Sarrat, M. Collard, P. Bertrand, T. CartierMichaud, P. Ghendrih, Y. Sarazin and X. Garbet, Global gyrokinetic simulations of TEM and TIM microturbulence, Phys. Plasmas 22, 082302 (2015)

[18] T. Drouot, E. Gravier, T. Reveille, M. Collard, Self-generated zonal flows in the plasma turbulence driven by trapped-ion and trapped-electron instabilities, Phys. Plasmas 22, 102309 (2015)

[19] A. Ghizzo, D. Del Sarto, X. Garbet, Y. Sarazin, Streamer-induced transport in the presence of trapped ion modes in tokamak plasmas, Phys. Plasmas 17, 092501 (2010)

[20] E. Sonnendrücker, J. Roche, P. Bertrand and A. Ghizzo, The Semi-Lagrangian Method for the Numerical Resolution of the Vlasov Equation, Journ. Comp. Phys. 149, 201 (1999)

[21] K. Pyragas, Continuous control of chaos by self-controlling feedback, Phys. Lett. A 170, $421(1992)$

[22] E. Gravier, X. Caron, G. Bonhomme and T. Pierre, Control of the chaotic regimes of nonlinear drift waves in a magnetized plasma, Phys. Plasmas 6, 1670 (1999)

[23] F. Palermo, X. Garbet, A. Ghizzo, T. Cartier-Michaud, P. Ghendrih, V. Grandgirard, Y. Sarazin, Shear flow instabilities induced by trapped ion modes in collisionless temperature gradient turbulence, Phys. Plasmas 22, 042304 (2015) 